\title{
In situ terahertz monitoring of an ice ball formation during tissue cryosurgery: a feasibility test
}

\author{
Arsen K. Zotov, ${ }^{\text {a Arsenii A. Gavdush, }}{ }^{\text {b }}$ Gleb M. Katyba $\odot,{ }^{\text {a,c }}$ \\ Larisa P. Safonova, ${ }^{c}$ Nikita V. Chernomyrdin $\odot,{ }^{b, c, d}$ and \\ Irina N. Dolganova ${ }^{\text {a,c,d, } * *}$ \\ ${ }^{a}$ Institute of Solid State Physics of the Russian Academy of Sciences, Chernogolovka, Russia \\ ${ }^{b}$ Prokhorov General Physics Institute of the Russian Academy of Sciences, Moscow, Russia \\ ${ }^{\mathrm{c}}$ Bauman Moscow State Technical University, Moscow, Russia \\ ${ }^{\mathrm{d} S e c h e n o v}$ First Moscow State Medical University (Sechenov University), \\ Institute for Regenerative Medicine, Moscow, Russia
}

\begin{abstract}
Significance: Uncontrolled cryoablation of tissues is a strong reason limiting the wide application of cryosurgery and cryotherapy due to the certain risks of unpredicted damaging of healthy tissues. The existing guiding techniques are unable to be applied in situ or provide insufficient spatial resolution. Terahertz (THz) pulsed spectroscopy (TPS) based on sensitivity of THz time-domain signal to changes of tissue properties caused by freezing could form the basis of an instrument for observation of the ice ball formation.

Aim: The ability of TPS for in situ monitoring of tissue freezing depth is studied experimentally. Approach: A THz pulsed spectrometer operated in reflection mode and equipped with a cooled sample holder and ex vivo samples of bovine visceral adipose tissue is applied. Signal spectrograms are used to analyze the changes of $\mathrm{THz}$ time-domain signals caused by the interface between frozen and unfrozen tissue parts.

Results: Experimental observation of TPS signals reflected from freezing tissue demonstrates the feasibility of TPS to detect ice ball formation up to $657-\mu \mathrm{m}$ depth.

Conclusions: TPS could become the promising instrument for in situ control of cryoablation, enabling observation of the freezing front propagation, which could find applications in various fields of oncology, regenerative medicine, and $\mathrm{THz}$ biophotonics.

(C) The Authors. Published by SPIE under a Creative Commons Attribution 4.0 Unported License. Distribution or reproduction of this work in whole or in part requires full attribution of the original publication, including its DOI. [DOI: 10.1117/1.JBO.26.4.043003]
\end{abstract}

Keywords: cryoablation; cryotherapy; terahertz biophotonics; tetrahertz pulsed spectroscopy; ice ball formation.

Paper 200372SSR received Nov. 15, 2020; accepted for publication Jan. 7, 2021; published online Jan. 27, 2021.

\section{Introduction}

Terahertz (THz) pulsed spectroscopy (TPS) is known as a promising tool of biophotonics and medical diagnosis. ${ }^{1-4}$ It demonstrates strong potential of study and differentiate various conditions of tissues, both ex vivo and in vivo, ${ }^{5-9}$ and imaging and characterization of neoplasms in different localizations, ${ }^{10-14}$ by means of endogenous markers of pathology, such as water content. However, high concentration of free and bound water in tissues restricts the penetration depth of $\mathrm{THz}$ waves by only several hundreds of microns. ${ }^{1}$ To overcome this limitation, different approaches can be applied. Among them are such techniques as tissue dehydration, ${ }^{15}$ paraffinembedding, ${ }^{16}$ lyophilization, ${ }^{17}$ compression, ${ }^{18}$ immersion optical clearing, ${ }^{19,20}$ and freezing. ${ }^{21-24}$ It was demonstrated that the latter one helps to increase the THz-wave penetration up to $1 \mathrm{~mm}$, changing at the same time dielectric properties of tissues in $\mathrm{THz}$ range. ${ }^{23}$ Along with reduction of

*Address all correpsondence to Irina N. Dolganova, dolganova@issp.ac.ru 
absorption, low temperatures alter the refractive index within certain limits, thus, yielding the dielectric contrast between tissues in different states. This opens further horizons of TPS applications, in particular, for monitoring of freezing depth in tissues during their cryosurgery.

Uncontrolled cryoablation of living tissues is a severe problem of modern cryosurgery, i.e., application of extreme cold aimed at rapid freezing and thus destroying or renewal of pathological tissues. ${ }^{25-29}$ Unless cryoablation possesses several advantages, such as relative painlessness, hemostatic effect, short recovering of patients, and immunostimulating effect, ${ }^{25,30,31}$ it is associated with certain risks of damaging healthy tissues surrounding the pathology and with possibility of incomplete cell death. ${ }^{28}$ In particular, the use of cryosurgery for the treatment of brain tumors and epilepsy is currently limited by the possible risks for the patient. ${ }^{32-34}$ Such guiding techniques as ultrasonography, ${ }^{35,36}$ magnetic resonance, and computer imaging ${ }^{37-42}$ do not enable the monitoring of the tissue freezing in situ with high spatial resolution near the cryoprobe. Alternative approach is based on measuring of tissue temperature using thermocouples (TCs) combined with cryoprobe. ${ }^{43}$ However, it allows controlling of the tissue condition only at the cryoprobe-tissue interface. Therefore, cryosurgery still needs guiding approaches for the efficient control of ice ball formation.

In this work, we study the ability of TPS to solve this problem. We experimentally demonstrate the changes of $\mathrm{THz}$ time-domain signal during tissue freezing, using ex vivo bovine adipose specimen and laboratory spectrometer equipped with special sample holder. Processing of $\mathrm{THz}$ pulsed signal within $45 \mathrm{~s}$ demonstrates the ability to detect the movement of freezing front up to the depth of $657 \mu \mathrm{m}$. The results of this work reveal the potential of TPS to control the initial stage of ice ball formation during tissue cryoablation.

\section{Experimental Setup}

To experimentally test the feasibility of TPS to detect the freezing depth in biological tissues, we use a laboratory THz pulsed spectrometer, which was described in detail in Ref. 44. It operates in a reflection mode and has a maximum spectral operating range from about 0.05 to $4.0 \mathrm{THz}$ and a maximum spectral resolution of about $0.002 \mathrm{THz}$. The $\mathrm{THz}$ wave features s-polarization. To avoid the impact of water vapors on the measurements, the spectrometer is purged with nitrogen gas. It is also equipped with a sample holder (Fig. 1). The latter consists of a reference window

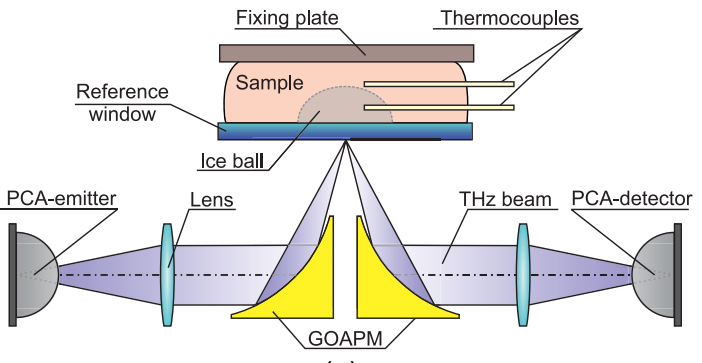

(a)

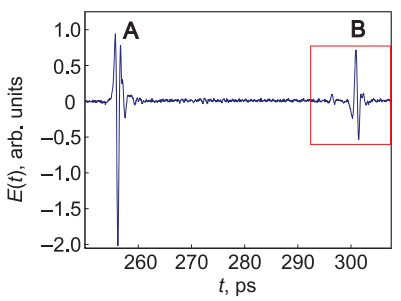

(c)

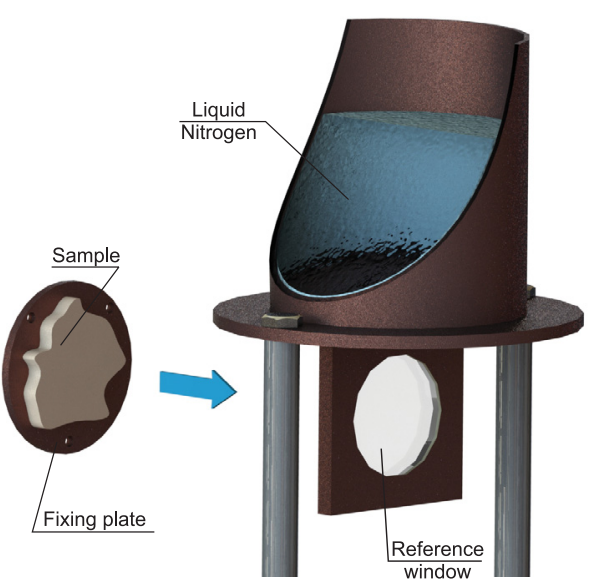

(b)

Fig. 1 Schematic of the ex vivo TPS measurement of the tissue freezing: (a) a reflection-mode measurement unit of the THz pulsed spectrometer, PCA stands for a photoconductive antenna, GOAPM stands for a gold off-axis parabolic mirror; (b) a sample holder; (c) an example of the sample time-domain signal $E(t)$ of the $\mathrm{THz}$ pulsed spectrometer, where $\mathrm{A}$ and $\mathrm{B}$ stand for pulses reflected from the interfaces free space-reference window and reference window-sample, respectively. 
made of sapphire, which on the one hand is placed on the $\mathrm{THz}$ beam path in contact with the tissue sample [see Fig. 1(a)]. On the other hand, it is inserted in the metal frame connected with the reservoir filled with liquid nitrogen prior to the measurements [Fig. 1(b)]. Thus, due to high thermal conductivity of metal and sapphire, the window possesses low and stable temperature during the measurements.

As a tissue sample, we use ex vivo bovine visceral adipose tissue because of its relatively homogeneous structure. The sample thickness is near $30 \mathrm{~mm}$ enabling prolonged duration of the freezing process. By two off-axis parabolic mirrors, the incident $\mathrm{THz}$ beam is focused on the interface between the reference window and the tissue; the reflected beam is collimated to the detector. Generation and detection of $\mathrm{THz}$ pulses are provided by two photoconductive antennas (PCAs). A typical form of the reflected THz time-domain signal is shown in Fig. 1(c). The sequence of pulses includes signal (A) reflected from free space-window interface and signal (B) reflected from the window-sample interface. Since we are interested in the latter one, the scanning range of the spectrometer is adjusted at this pulse (marked with red rectangle).

The measurements are performed at room temperature $24^{\circ} \mathrm{C}$. However, the sample is preliminary heated to the temperature $37^{\circ} \mathrm{C}$ aimed at the slowing down the initial stage of freezing. After the contact between the tissue and the cooled window, $\mathrm{THz}$ pulsed signal is constantly recorded with the period of $15 \mathrm{~s}$ until the sample is completely frozen. For additional control of sample freezing, its temperature is measured by the inserted TCs.

\section{THz Pulsed Spectroscopy of a Freezing Tissue}

\subsection{THz Dielectric Properties of a Sample}

The sample refractive index was characterized before and after freezing. For this purpose, the complex amplitude of the incident THz field is considered to have the form $E(t)=A_{0} \exp (i \phi)$, where $A_{0}$ and $\phi$ are the amplitude and phase of the THz field, respectively. Assuming the negligible losses in the reference window and the normal incidence of $\mathrm{THz}$ beam, the signal amplitudes $A_{1}$ and $A_{2}$ of pulses reflected from the first and second interfaces [see wavelets (A) and (B) in Fig. 1(c)] are described by the Fresnel coefficients

$$
\begin{gathered}
A_{1}=R_{01} A_{0}, \\
A_{2}=T_{01} R_{12} T_{10} A_{0},
\end{gathered}
$$

where $R_{m k}$ and $T_{m k}$ are the Fresnel coefficients of amplitude reflection and transmission between $m$ 'th and $k$ 'th media, which for s-polarized wave are determined by the corresponding refractive indices $n_{m}$ and $n_{k}$

$$
\begin{aligned}
& R_{m k}=\frac{n_{m}-n_{k}}{n_{m}+n_{k}}, \\
& T_{m k}=\frac{2 n_{k}}{n_{m}+n_{k}} .
\end{aligned}
$$

In this work, refractive indices for free space and reference window are $n_{0}=1$ and $n_{1}=3.07$, respectively, at $1-\mathrm{THz}$ frequency. ${ }^{45}$ Estimating the relation $\left|A_{2} / A_{1}\right|$ from the measured TPS waveforms and accounting the dispersion relation, the values of the sample refractive index $n_{2}$ at 1-THz frequency were determined for two conditions of the tissue, i.e., $n_{R}=1.84 \pm$ 0.01 at room temperature and $n_{F}=1.37 \pm 0.01$ after freezing. Thus, freezing of the adipose tissue leads to significant changes of its refractive index. The value $n_{F}$ is used further for estimation of the freezing depth $z$ according to the relation $z=t c /\left(2 n_{F}\right)$, where $t$ is the temporal position of the detected freezing depth, $c$ is speed of light in free space. 


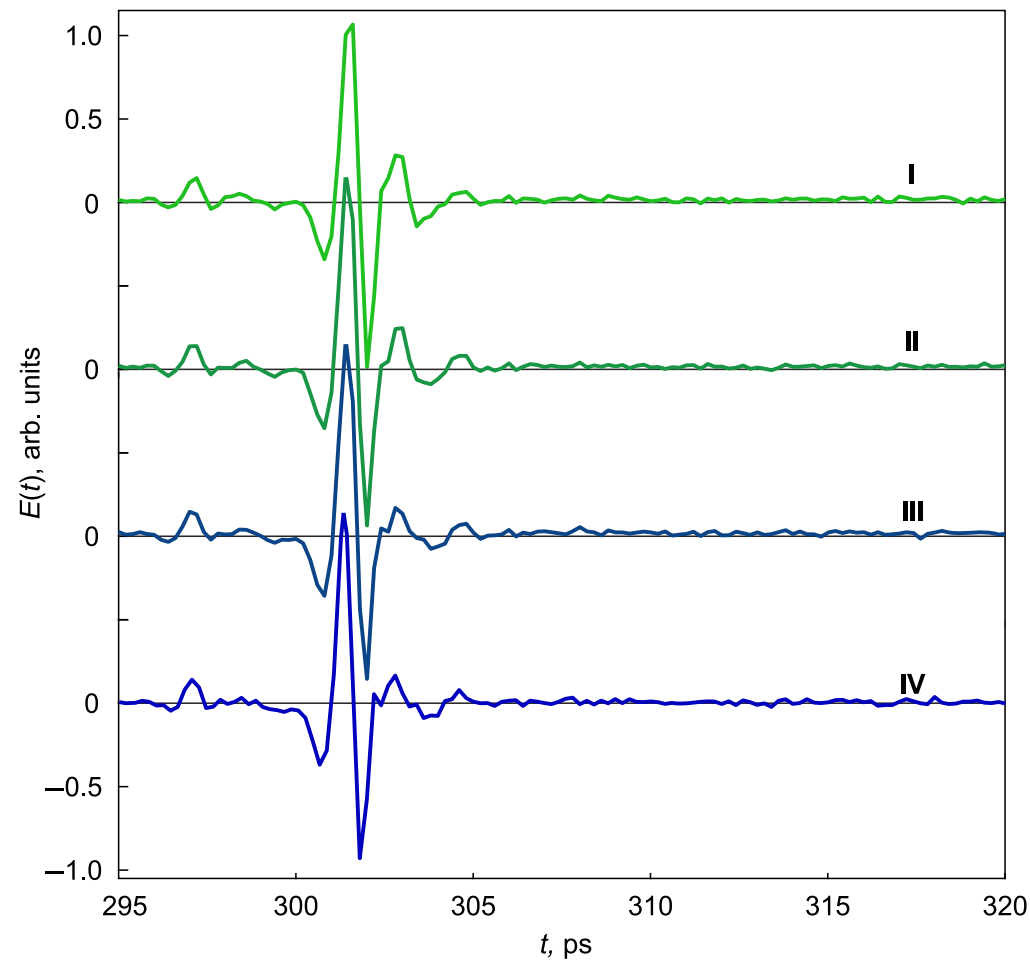

Fig. 2 The waterfall of $\mathrm{THz}$ time-domain signals reflected from the window-tissue interface and detected by the spectrometer during the tissue freezing. Signals I, II, III, and IV correspond to $t=0,15,30$, and $45 \mathrm{~s}$ moments of freezing, respectively.

\subsection{Analysis of THz Signal Spectrograms}

According to the measurement of sample temperature, the freezing depth of $4 \mathrm{~mm}$ was reached in $<1 \mathrm{~min}$. This depth significantly exceeds the typical values of $\mathrm{THz}$ penetration depth in frozen biological tissues. ${ }^{23}$ Thus, only several $\mathrm{THz}$ waveforms can be recorded during this stage of the analyzed process. To increase the number of detected signals, the accumulation time and discretization of the spectrometer should be reduced.

Figure 2 shows the sequence of THz waveforms detected at (I) $0 \mathrm{~s}$, (II) $15 \mathrm{~s}$, (III) $30 \mathrm{~s}$, and (IV) $45 \mathrm{~s}$ from the beginning of the sample cooling. At the initial moment $t=0$ of the ice front propagation, the temporal width of first $\mathrm{THz}$ pulse, caused by the window-tissue interface, is about $5 \mathrm{ps}$, which corresponds to $\sim 0.5 \mathrm{~mm}$ of the sample depth. Within this tissue layer, the second pulse caused by the existence of the ice front interface, is probably overlapped by the first one. Its amplitude is expected to be significantly smaller, since the dielectric contrast between frozen and unfrozen tissue is lower than that for the tissue and the reference window. Thus, it is difficult to estimate directly from the $\mathrm{THz}$ waveforms the moving position of the second pulse.

To overcome this problem, we analyze the signal intensity spectrograms in addition to their temporal waveforms. Figures 3(a)-3(d) show the spectrograms of the measured signals shown in the insets [Figs. 3(e)-3(h)]. Comparing them between each other, it is possible to detect the temporal position of the pulse reflected from the ice front. Figures 3(i)-3(k) show the difference $\Delta I(t)$ between the normalized spectrogram $I_{n}(t)=I(t) / \max [I(t)]$ at $t=15,30$, and $45 \mathrm{~s}$ and the initial normalized spectrogram at $t=0$. Obviously, the first pulse is altering during the first $30 \mathrm{~s}$, then its appearance remains stable. We can clearly observe the presence of the second pulse at $t=15$ and $30 \mathrm{~s}$, which indicates the freezing depths of $z=328 \mu \mathrm{m}$ and $z=657 \mu \mathrm{m}$, respectively; then, its amplitude becomes lower due to absorbtion, which does not allow us to detect it. Therefore, during $30 \mathrm{~s}$ starting from the tissue cooling, TPS provides an ability to analyze the ice ball formation and estimate the freezing depth. It should be mentioned that time-domain $\mathrm{THz}$ signals feature rather weak changes at the determined moments, not allowing us to evaluate them directly. To demonstrate this, the detected positions of the second pulses at $t=15$ and $30 \mathrm{~s}$ are 

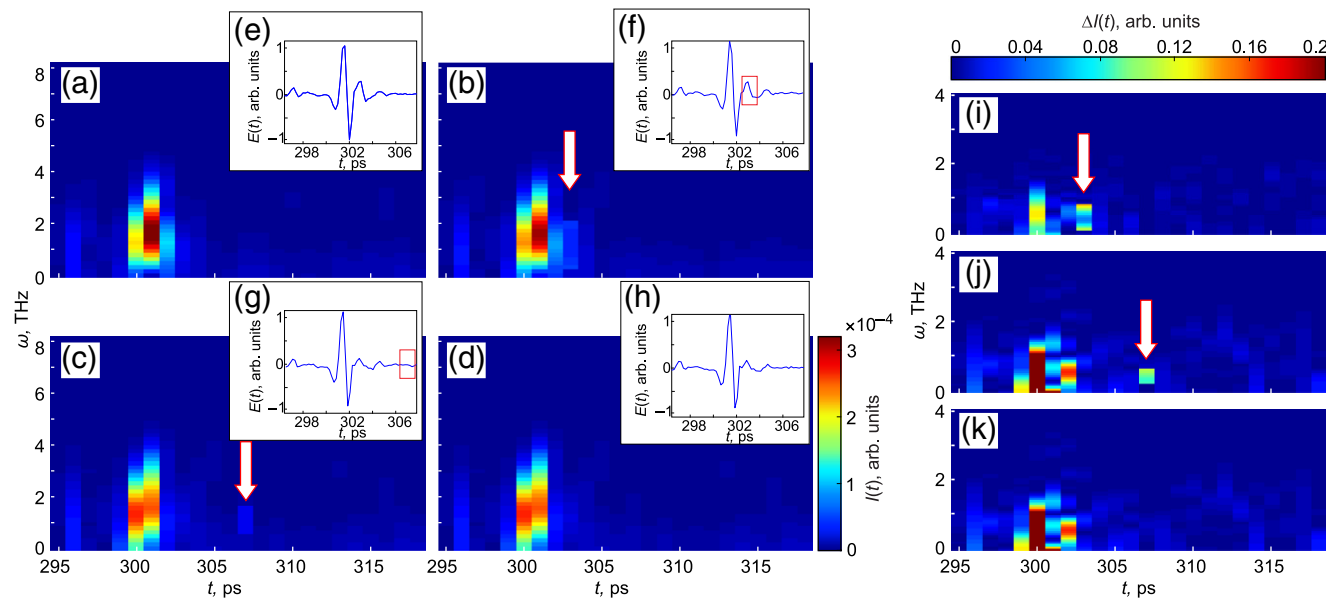

Fig. 3 Analysis of $\mathrm{THz}$ time-domain signals detected during the tissue freezing. (a)-(d) Signal intensity spectrograms, where white arrows indicate the positions of second pulses reflected from the freezing front; (e) and (f) the temporal amplitudes of the signals, where red rectangles mark the same positions; (a) and (e) correspond to the moment $t=0$; (b) and (f) $15 \mathrm{~s}$; (c) and (g) $30 \mathrm{~s}$; and (d) and (h) $45 \mathrm{~s}$; (i)-(k) the differences $\Delta I(t)$ between the normalized spectrogram $I_{n}(t)=$ $I(t) / \max [I(t)]$ at $t=15,30$, and $45 \mathrm{~s}$ and the initial normalized spectrogram at $t=0$.

marked with red rectangles on the THz waveforms [see Figs. 3(f) and 3(g)]. Due to the absence of second pulses at $t=0$ and $45 \mathrm{~s}$, there are no marks on Figs. 3(e) and 3(h).

\section{Results and Discussion}

The estimation of the tissue freezing depth is shown in Fig. 4, where the values obtained by TPS are combined with the direct measurements of the sample temperature. Figure 4(b) demonstrates the temperature change at two depths 4 and $11 \mathrm{~mm}$ from the window-tissue interface. The slowdown of the freezing process at the beginning can be explained by the initial cooling of the tissue from $37^{\circ} \mathrm{C}$ to $0^{\circ} \mathrm{C}$. In our feasibility study, the maximal freezing depth observed by TPS is limited by the value $657 \mu \mathrm{m}$. However, we believe that it could be increased using THz time-domain

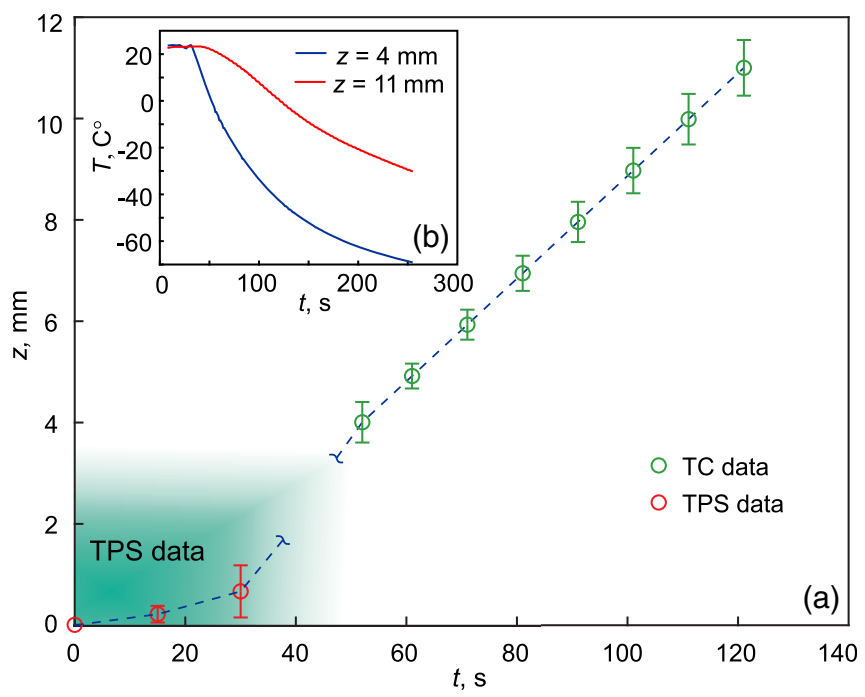

Fig. 4 An estimation of the tissue freezing depth. (a) The combination of TPS measurements (points marked with red color) and data obtained by TCs (points marked with green color), here, TC stands for thermocouple; (b) temperature changes of the freezing tissue at depths of 4 and $11 \mathrm{~mm}$ from the reference window. Error bars represent $\pm 2 \sigma$ confidential interval of measurements. 
spectrometer with more efficient performance, particularly, scanning rate, which would yield the reduction of the detection period and increasing of the temporal resolution.

Though the penetration depth of THz waves even in frozen tissue remains extremely low, the detection of the freezing depth by TPS could provide rather high precision of monitoring ice front propagation, enabling the observation and control of cryoablation and cooling of cell and artificial tissue structures, and agglomeration of cell spheroids. Thus, it could become a beneficial research instrument in regenerative medicine and microbiology.

$\mathrm{THz}$ technologies are still far from clinical application, suffering from a list of problems, such as low performance of $\mathrm{THz}$ sources and detectors, and low spatial resolution due to high absorbtion and scattering. ${ }^{46,47}$ However, the rapid progress of THz sources and optical components in the recent years ${ }^{48-52}$ stimulates the further improvements of $\mathrm{THz}$ instruments for biophotonics. A strong limitation is caused by the absence of efficient waveguides and fibers. ${ }^{53}$ Nevertheless, the sapphire shaped crystal waveguides, exploiting both anti-resonant reflecting optical and photonic crystal waveguidance principles, have certain perspectives for delivering $\mathrm{THz}$ pulses with relatively low losses and dispersion; ${ }^{44,54}$ thus, they could form the basis for further developments of $\mathrm{THz}$ instruments for biophotonics and medicine. In addition, sapphire features high thermal conductivity at cryogenic temperatures, being more efficient material for cryosurgery than metal (copper, titanium nickelide, and stainless steel), since it enables higher tissue freezing rate. ${ }^{53}$ Combination of these advantages opens future perspectives for the appearance of sapphire probes for controlled cryoablation.

Cryosurgery demonstrates high potential in oncology, in particular, to remove neoplasms and metastases of different localizations, such as liver, ${ }^{37}$ breast, ${ }^{55}$ lungs, ${ }^{40}$ prostate,${ }^{56}$ kidney, ${ }^{57}$ stomach, ${ }^{58}$ and skin. ${ }^{59}$ Considering the ability of $\mathrm{THz}$ technologies for tumor diagnosis along with currently developing $\mathrm{THz}$ instruments and methods for healthy and pathological tissues differentiation, ${ }^{2}$ the combination of $\mathrm{THz}$ preoperative characterization of tissue with further TPS-controlled cryoablation could become an effective tool for oncology.

It is worth mentioning that several techniques enable increasing of low penetration depth of $\mathrm{THz}$ waves into living tissues. Among them are methods of immersion optical clearing, ${ }^{19}$ which could also be combined with $\mathrm{THz}$ freezing depth detection. However, a list of hyperosmotic agents applied for tissue clearing act as cryoprotectors (e.g., glycerol, dimethyl sulfoxide, and propylene glycol), since they substitute the interstitial water. Thus, the particular approaches of their application for increasing of THz-wave penetration into tissues during their freezing, such as concentration of aqueous solutions and exposure time, are the subjects of further studies.

\section{Conclusion}

The performed experimental study demonstrates the feasibility of in situ observation of the ice ball formation in tissues using TPS instrumentation. Applying the spectrogram analysis of THz time-domain signals recorded in the reflection-mode of $\mathrm{THz}$ pulsed spectrometer during tissue freezing, it is possible to detect the positions of the freezing depth up to $657 \mu \mathrm{m}$. We believe that the described approach would be helpful for the development of novel approaches and instruments of controlled cryoablation and would be useful for various tasks of oncology, regenerative medicine, microbiology, and further progress of $\mathrm{THz}$ biophotonics.

\section{Disclosures}

The authors declare no conflict of interest.

\section{Acknowledgments}

This work was supported by the Russian Science Foundation (RSF), Project No. \#19-79-10212.

\section{Code, Data, and Materials Availability}

All data generated or analyzed during this study are included in this article. 


\section{References}

1. O. A. Smolyanskaya et al., "Terahertz biophotonics as a tool for studies of dielectric and spectral properties of biological tissues and liquids," Prog. Quantum Electron. 62, 1-77 (2018).

2. K. I. Zaytsev et al., "The progress and perspectives of terahertz technology for diagnosis of neoplasms: a review," J. Opt. 22(1), 013001 (2020).

3. J.-H. Son, S. J. Oh, and H. Cheon, "Potential clinical applications of terahertz radiation," J. Appl. Phys. 125(19), 190901 (2019).

4. H. Cheon, H. Yang, and J. Son, "Toward clinical cancer imaging using terahertz spectroscopy," IEEE J. Sel. Top. Quantum Electron. 23(4), 1-9 (2017).

5. J. Wang et al., "In vivo terahertz imaging to evaluate scar treatment strategies: silicone gel sheeting," Biomed. Opt. Express 10(7), 3584-3590 (2019).

6. G. Hernandez-Cardoso et al., "Terahertz imaging for early screening of diabetic foot syndrome: a proof of concept," Sci. Rep. 7, 42124 (2017).

7. N. Bajwa et al., "Non-invasive terahertz imaging of tissue water content for flap viability assessment," Biomed. Opt. Express 8(1), 460-474 (2017).

8. Y. Cao et al., "Qualitative and quantitative detection of liver injury with terahertz timedomain spectroscopy," Biomed. Opt. Express 11(2), 982-993 (2020).

9. E. P. Parrott et al., "Terahertz pulsed imaging in vivo: measurements and processing methods," J. Biomed. Opt. 16(10), 106010 (2011).

10. $\mathrm{D}$. $\mathrm{Li}$ et al., "Detecting melanoma with a terahertz spectroscopy imaging technique," Spectrochim. Acta A 234, 118229 (2020).

11. S. Oh et al., "Study of freshly excised brain tissues using terahertz imaging," Biomed. Opt. Express 5(8), 2837-2842 (2014).

12. A. Gavdush et al., "Terahertz spectroscopy of gelatin-embedded human brain gliomas of different grades: a road toward intraoperative THz diagnosis," J. Biomed. Opt. 24(2), 027001 (2019).

13. P. C. Ashworth et al., "Terahertz pulsed spectroscopy of freshly excised human breast cancer," Opt. Express 17(15), 12444-12454 (2009).

14. K. I. Zaytsev et al., "Highly accurate in vivo terahertz spectroscopy of healthy skin: variation of refractive index and absorption coefficient along the human body," IEEE Trans. Terahertz Sci. Technol. 5(5), 817-827 (2015).

15. Y. He et al., "Determination of terahertz permittivity of dehydrated biological samples," Phys. Med. Biol. 62(23), 8882-8893 (2017).

16. K. Meng et al., "Terahertz pulsed spectroscopy of paraffin-embedded brain glioma," J. Biomed. Opt. 19(7), 077001 (2014).

17. G. Png et al., "The impact of hydration changes in fresh bio-tissue on $\mathrm{THz}$ spectroscopic measurements," Phys. Med. Biol. 53(13), 3501-3517 (2008).

18. J. Wang et al., "THz in vivo measurements: the effects of pressure on skin reflectivity," Biomed. Opt. Express 9(12), 6467-6476 (2018).

19. G. R. Musina et al., "Optimal hyperosmotic agents for tissue immersion optical clearing in terahertz biophotonics," J. Biophotonics 13(12), e202000297 (2020).

20. A. Kolesnikov et al., "THz monitoring of the dehydration of biological tissues affected by hyperosmotic agents," Phys. Wave Phenom. 22, 169-176 (2014).

21. H. Hoshina et al., "Terahertz pulsed imaging of frozen biological tissues," Appl. Phys. Lett. 94(12), 123901 (2009).

22. Y. Sim et al., "Temperature-dependent terahertz imaging of excised oral malignant melanoma," IEEE J. Biomed. Health 17(4), 779-784 (2013).

23. Y. C. Sim et al., "Terahertz imaging of excised oral cancer at frozen temperature," Biomed. Opt. Express 4(8), 1413-1421 (2013).

24. J. Y. Park et al., "Terahertz imaging of metastatic lymph nodes using spectroscopic integration technique," Biomed. Opt. Express 8(2), 1122-1129 (2017).

25. E. Vansonnenberg et al., Tumor Ablation: Principles and Practice, Springer-Verlag, New York (2005).

26. P. Mazur, "Cryobiology: the freezing of biological systems," Science 168(3934), 939-949 (1970). 
27. A. A. Gage and J. Baust, "Mechanisms of tissue injury in cryosurgery," Cryobiology 37(3), 171-186 (1998).

28. J. Baust et al., "Mechanisms of cryoablation: clinical consequences on malignant tumors," Cryobiology 68(1), 1-11 (2014).

29. N. Korpan, Basics of Cryosurgery, Springer-Verlag, Wien (2001).

30. K. F. Chu and D. E. Dupuy, "Thermal ablation of tumours: biological mechanisms and advances in therapy," Nat. Rev. Cancer 14(3), 199-208 (2014).

31. F. Yuan, G. Zhao, and F. Panhwar, "Enhanced killing of HepG2 during cryosurgery with Fe3O4-nanoparticle improved intracellular ice formation and cell dehydration," Oncotarget 8(54), 92561-92577 (2017).

32. T. Tokiwa et al., "A palm-sized cryoprobe system with a built-in thermocouple and its application in an animal model of epilepsy," IEEE Trans. Biomed. Eng. 66(11), 3168-3175 (2019).

33. C. Li et al., "MR imaging-guided cryoablation of metastatic brain tumours: initial experience in six patients," Eur. Radiol. 20(2), 404-409 (2010).

34. B. Martynov et al., "Stereotactic cryodestruction of gliomas," Prog. Neurol. Surg. 32, 27-38 (2018).

35. I. G. Berim et al., "Radial probe ultrasound-guided cryobiopsy," J. Bronchol. Interventional Pulmonol. 24(2), 170-173 (2017).

36. M. Ahmed et al., "Percutaneous ultrasound-guided cryoablation for symptomatic plantar fibromas," CardioVasc. Interventional Radiol. 41, 298-304 (2018).

37. K. Zhou et al., "Combined magnetic resonance imaging and optical surgical navigation system guidance of percutaneous liver cryoablation in a porcine model," Am. J. Transl. Res. 10(5), 1531-1538 (2018).

38. J. Lichter et al., "Real-time magnetic resonance imaging-guided cryoablation of the pulmonary veins with acute freeze-zone and chronic lesion assessment," EP Europace 21(1), 154-162 (2019).

39. W. Fan et al., "Percutaneous computed tomography-guided cryoablation for recurrent retroperitoneal soft tissue sarcoma: a study of safety and efficacy," Oncotarget 7(27), 42639-42649 (2016).

40. C. Prud'homme et al., "Image-guided lung metastasis ablation: a literature review," Int. J. Hyperth. 36(2), 37-45 (2019).

41. J. Pohlan et al., "Computed tomography thermography for ablation zone prediction in microwave ablation and cryoablation: advantages and challenges in an ex vivo porcine liver model," J. Comput. Assist. Tomogr. 44(5), 744-749 (2020).

42. Y. Yang et al., "Retrospective analysis of CT-guided percutaneous cryoablation for treatment of painful osteolytic bone metastasis," Cryobiology 92, 203-207 (2020).

43. M. Shurrab et al., "The cooling performance of a cryoprobe: establishing guidelines for the safety margins in cryosurgery," Int. J. Refrig. 67, 308-318 (2016).

44. G. M. Katyba et al., "Sapphire photonic crystal waveguides for terahertz sensing in aggressive environments," Adv. Opt. Mater. 6(22), 1800573 (2018).

45. D. Grischkowsky et al., "Far-infrared time-domain spectroscopy with terahertz beams of dielectrics and semiconductors," J. Opt. Soc. Am. B 7, 2006-2015 (1990).

46. I. N. Dolganova et al., "The role of scattering in quasi-ordered structures for terahertz imaging: local order can increase an image quality," IEEE Trans. Terahertz Sci. Technol. 8(4), 403-409 (2018).

47. K. I. Zaytsev et al., "Overcoming the Abbe diffraction limit using a bundle of metal-coated high-refractive-index sapphire optical fibers," Adv. Opt. Mater. 8(18), 2000307 (2020).

48. D. V. Lavrukhin et al., "Terahertz photoconductive emitter with dielectric-embedded highaspect-ratio plasmonic grating for operation with low-power optical pumps," AIP Adv. 9(1), 015112 (2019).

49. D. V. Lavrukhin et al., "Shaping the spectrum of terahertz photoconductive antenna by frequency-dependent impedance modulation," Semicond. Sci. Technol. 34, 034005 (2019).

50. D. S. Ponomarev et al., "Enhanced terahertz emission from strain-induced InGaAs/InAlAs superlattices," J. Appl. Phys. 125(15), 151605 (2019). 
51. V. E. Ulitko et al., "Nanoporous $\mathrm{SiO} 2$ based on annealed artificial opals as a favorable material platform of terahertz optics," Opt. Mater. Express 10(9), 2100-2113 (2020).

52. A. A. Gavdush et al., "Proof of concept for continuously-tunable terahertz bandpass filter based on a gradient metal-hole array," Opt. Express 28(18), 26228-26238 (2020).

53. G. Katyba et al., "Sapphire shaped crystals for waveguiding, sensing and exposure applications," Prog. Cryst. Growth Charact. Mater. 64(4), 133-151 (2018).

54. K. I. Zaytsev et al., "Terahertz photonic crystal waveguides based on sapphire shaped crystals," IEEE Trans. Terahertz Sci. Technol. 6(4), 576-582 (2016).

55. Z. Habrawi et al., "Cryoablation: a promising non-operative therapy for low-risk breast cancer," Am. J. Surg. 221, 127-133 (2021).

56. K. Kinsman et al., "Whole-gland prostate cancer cryoablation with magnetic resonance imaging guidance: one-year follow-up," CardioVasc. Interventional Radiol. 41, 344-349 (2018).

57. S. Hebbadj et al., "Safety considerations and local tumor control following percutaneous image-guided cryoablation of T1b renal tumors," CardioVasc. Interventional Radiol. 41, 449-458 (2018).

58. W. Zheng et al., "A novel endoscopic visible full-thickness cryoablation device on stomach," Sci. Rep. 10, 5203 (2020).

59. G. Mpourazanis et al., "The effectiveness of photodynamic therapy and cryotherapy on patients with basal cell carcinoma: a systematic review and meta-analysis," Dermatologic Therapy 33, e13881 (2020).

Biographies of the authors are not available. 